\title{
La signalisation hormonale dans la résistance des plantes aux bioagresseurs
}

\author{
Aida Jalloul ${ }^{1,2}$ \\ Alain Clerivet ${ }^{1,2}$ \\ Michel Nicole ${ }^{1,2}$ \\ ${ }^{1}$ Faculté d'agronomie de Damas \\ BP 30621 Damas \\ Syrie \\ 2 IRD UMR RPB Résistance des plantes \\ IRD-Cirad-UM2 \\ BP 64501 \\ 34394 Montpellier Cedex 5 \\ France \\ <aida-jal@scs-net.org> \\ <aclerivet@iutmontp.univ-montp2.fr> \\ $<$ nicole@mpl.ird.fr>
}

\begin{abstract}
Résumé
Pour assurer sa survie face aux stress biotiques, la plante met en place différentes stratégies de défense. Suivant la biologie du parasite et la nature du parasitisme, elle adaptera ses réponses par une régulation fine de ses mécanismes de défense sous le contrôle de ses hormones dont elle va orienter les fonctions. L'acide salicylique, l'acide jasmonique et l'éthylène pilotent des voies de signalisation dont le rôle dans la résistance est le mieux établi. Mais d'autres phytohormones, comme l'acide abscissique, occupent une place prépondérante dans la régulation de la défense des plantes. L'interconnection et la coordination de ces voies de signalisation sont des éléments clés pour une expression optimisée de la résistance des plantes aux bioagressseurs.
\end{abstract}

Mots clés : hormone végétale ; résistance des plantes ; stress biotique.

Thèmes : productions végétales ; pathologie.

\section{Abstract \\ Hormone signalling in plant resistance}

Plant survival to biotic stresses results from various defence strategies. Depending on the pathogen and nature of the parasitism, a plant will adapt its responses through a fine-tuning regulation of defence mechanisms under the control of its hormones whose functions will be reoriented. Salicylic acid, jasmonic acid and ethylene drive signalling pathways, the role of which is well established in plant resistance. However, additional hormones, including abscisic acid, seem to have an essential role in plant defence regulation. Crosstalk and coordination of these signalling pathways are key elements for the expression of optimized plant resistance to pathogens.

Key words: biotic stress; plant hormones; plant resistance.

Subjects: pathology; vegetal productions.
$\mathrm{L}^{\prime}$ une des caractéristiques de la résistance des plantes aux agents biotiques est la capacité à induire des réactions de défense au site d'infection (résistance spécifique et résistance partielle ; Pontier et al., 1998) et dans des zones éloignées du point d'infection, au sein de tissus a priori non infectés (résistance systémique acquise [SAR] et résistance systémique induite [ISR]) (Vallad et Goodman, 2004). Dans ce dernier cas, la notion de systémie implique l'existence d'un signal qui va commander l'activation ou l'amplification de réactions de défense sur de longues distances (Heil et Ton, 2008). On parlera alors de voie de signalisation, dont le concept a été cerné dans les années 1990. Un signal va intégrer dans la cellule une information extracellulaire dans le but de déclencher un mécanisme dont l'aboutissement est l'expression d'un évènement ciblé, comme, par exemple, les réponses de défense. Chez les plantes, les hormones jouent le rôle de "molécules signals ", ou messagers, en régulant l'activité cellulaire dont elles modifient le comportement durant les interactions avec un parasite. Peu de données ont été acquises quant au déterminisme génétique de la régulation hormonale, mais on soupçonne qu'un nombre très réduit de gènes ont pour rôle, plutôt d'informer la plante du moment et du lieu où produire une hormone lors d'une étape clef de sa vie 
(Stepanova et al., 2008). L'examen de la littérature révèle une abondance de références concernant trois hormones aux fonctions importantes dans l'installation des résistances: l'acide salicylique (AS), l'acide jasmonique (AJ) et l'éthylène (ET). Mais, depuis quelques années, l'intérêt se porte vers d'autres hormones, les gibbérélinnes, les auxines, les brassinostéroides et, surtout, l'acide abscissique (ABA), qui semble avoir une place non négligeable dans la régulation de la défense chez les plantes. Dans cet article, des exemples illustreront l'influence et la spécificité de chacune de ces " molécules signals "; une place sera également réservée à la coordination de ces voies de signalisation, signalisation qui optimise les stratégies de défense, non seulement en termes d'efficacité, mais également en matière d'économie d'énergie.

\section{SAR : chasse gardée de l'acide salicylique}

Les travaux conduits sur la réaction d'hypersensibilité (RH) ont mis en exergue le déclenchement concomitant d'une réponse de type systémique étroitement liée à des augmentations de la teneur en AS, acide carboxylique (acide 2-hydroxybenzoïque) issu du métabolisme des phénylpronanoïdes. L'AS s'accumule, non seulement localement au site d'infection, mais également dans la plante entière, pour prémunir les tissus d'attaques parasitaires ultérieures quel que soit l'agent pathogène (Delannoy et al., 2005). Mais la production locale d'AS n'est pas nécessaire à son accumulation systémique. Plusieurs marqueurs sont associés à la SAR, dont la synthèse de protéines liées à la pathogenèse (PR-P) via la transcription du gène NPR1. La surexpression de ce gène chez Arabidopsis confère à cette plante une résistance à certaines souches virulentes de la bactérie du genre Pseudomonas. Les mécanismes de la transduction du signal de la voie de l'AS impliquent le choc oxydatif, l'activation de la cascade des MAP ( mitogen-activated protein) kinases et l'induction de gènes de défense. L'utilisation de plants transgéniques $N a G h$ de pommes de terre, incapables d'accumuler l'AS, a conforté ces observations (Halim et al., 2007).

La nature du signal qui va activer la synthèse d'AS à distance du site d'infection n'est cependant pas encore clairement élucidée (Vlot et al., 2008). Chez le tabac, des expérimentations récentes d'extinction de gènes ont démontré que la forme méthylée de l'AS (MeAS) serait la molécule de translocation. L'inhibition du gène codant pour la protéine qui convertit le MeAS en AS conduit en effet à une incapacité de la plante à exprimer la SAR (Park et al., 2007). Chez Arabidopsis, le scénario du transfert du signal serait plutôt associé à des dérivés de lipides chloroplastiques voire à l'AJ (Truman et al., 2007). Quoi qu'il en soit, le coût énergétique de l'activation de la SAR chez la plante serait compensé par un approvisionnement accru en énergie lumineuse via une régulation positive par les phytochromes (Griebel et Zeier, 2007).

\section{Omniprésence de la voie \\ de l'acide jasmonique dans la résistance}

L'AJ, octadécanoïde dérivé de l'oxydation d'un acide gras - l'oxylipine -, est une molécule majeure dans la vie de plante, puisqu'elle est engagée dans plusieurs étapes du développement. Cette hormone a longtemps été associée aux réactions de la plante induites suite à une blessure (Farmer et Ryan, 1992). L'implication de l'AJ dans l'ISR a ensuite été révélée lorsque des plantes en contact avec des rhizobactéries non pathogènes ont augmenté leur résistance globale vis-à-vis de certains micro-organismes pathogènes (van Loon et al., 1998). Enfin, plus récemment, des données tendent à attribuer à l'AJ un rôle prépondérant dans l'exécution de la mort cellulaire programmée liée à la RH. Ce sont, en fait, l'AJ, sa forme méthylée (MeAJ) et leurs dérivés - appelés jasmonates (JA) qui possèdent des propriétés bioactives potentielles dans la régulation de la défense aux stress biotiques (Kazan et Manners, 2008). Le développement, chez Arabidopsis, de mutants coi (coronative insensitive 1) - qui présentent des déficiences de certains mécanismes jasmonatedépendants : jar1 (jasmonate resistant 1) et jin1 (jasmonate insensitive1 - a permis de lever partiellement le voile sur la complexité de la voie de l'AJ. Les récepteurs cellulaires de l'AJ et de ses dérivés sont en fait très diversifiés, ce qui traduit le potentiel de réponse des plantes à différents stimuli endogènes ou exogènes. La voie de signalisation de l'AJ se distingue aussi par son aptitude à s'autoréguler; les gènes de la voie de biosynthèse de l'AJ peuvent ainsi être activés par les JA, ce qui suggère un rétrocontrôle positif impliquant, entre autres, les canaux calciques (Bonaventure et al., 2007). Malgré les progrès récents quant à la fonction de l'AJ dans la résistance des plantes aux agressions parasitaires, la connaissance des fondements moléculaires des mécanismes de régulation que contrôle cette molécule demeure encore très fragmentaire et constitue un terrain d'investigations très fertile (Katsir et al., 2008). Dans cette optique, l'analyse des mutants jaz (jasmonate ZIM domain: domaines codant des protéines qui répriment l'expression des gènes sensibles à l'AJ) permettra d'améliorer la compréhension du rôle de l'AJ.

\section{L'éthylène, un modulateur des interactions plantes-parasites}

L'ET est une hormone dont le rôle dans l'organogenèse végétale est bien établi. Cet hydrocarbure insaturé volatile $\left(\mathrm{C}_{2} \mathrm{H}_{4}\right)$, découvert en tant que phytohormone en 1901, est une molécule multifonctionnelle chez la plante. L'augmentation de sa production suite à une infection microbienne est rapide et précoce, associée à certaines réactions de défense (Boller, 1991). Elle peut être cependant très localisée et s'accumuler en grande quantité dans les cellules en contact avec l'agent pathogène. Mais elle a également été identifiée dans les plantes âgées, en relation avec la résistance liée à l'âge (Age-Related Resistance, ARR ; Panter et Jones, 2002). Le paradoxe de l'ET réside dans la dualité de son comportement au cours des interactions, modulant soit les mécanismes impliqués dans l'expression de la maladie, soit ceux contrôlant la résistance (van Loon et al., 2006). L'utilisation de mutants surproducteurs d'ET (eto) ou insensibles à cette molécule (ein), car affectés dans certains de ses récepteurs, a permis de découpler les fonctions respectives de l'hormone durant la relation de la plante avec son parasite (Broekaert et al., 2006). Le traitement de certaines espèces avec de l'ET accélère le processus de sénescence des 
tissus et accroît en parallèle leur sensibilité. En conditions contrôlées, un traitement préventif des plantes favorise la résistance alors qu'un traitement après infection va augmenter leur sensibilité. C'est, semblet-il, au niveau des récepteurs de l'ET que s'opère le déterminisme de sa fonction : limitation de la croissance du parasite, blocage de la propagation systémique du champignon, amplification de la virulence bactérienne - certains micro-organismes sécrètent de l'ET-, réduction ou accroissement des symptômes... D'une manière générale, chez Arabidopsis, l'ET et l'AJ contribuent à la résistance aux agents nécrotrophes, alors que la résistance induite par les biotrophes serait préférentiellement AS-dépendante ou AJ et ASdépendante (Thomma et al., 2001). Au cours de la résistance induite, l'ET est capable d'activer plusieurs réactions de défense comme l'accumulation de phytoalexines, de flavonoides ou de PR-P. La signification de la synthèse d'ET au cours de certaines RH est, en revanche, plus obscure; certains auteurs lui attribuent un rôle dans la taille des lésions et le transfert du signal dans l'installation de la SAR, voire dans la production locale de PR-P (Verberne et al., 2003). À la lumière de ces quelques observations, la place de l'ET dans la résistance apparaît plus diversifiée que celle d'autres hormones : son importance et sa finalité vont dépendre du type de parasite, de la nature du parasitisme et de la famille de plantes concernées (brassicacae, solanacae, etc.).

\section{L'ambition}

\section{de l'acide abscissique dans le contrôle de la résistance}

L'ABA est un sesquiterpène étroitement lié au développement de la plante; son implication dans la gestion des stress abiotiques est de même largement reconnue. Paradoxalement, son rôle dans la résistance des plantes aux infections a été comparativement négligé, même si pressenti dès les années 1980 (MauchMani et Boller, 2005). L'action de l'ABA au cours des interactions se partage entre l'accroissement de la sensibilité par la suppression de l'accumulation de phytoalexines via l'inhibition de la PAL et son influence positive sur la résistance en par- ticipant au renforcement des parois (Flors et al., 2005). En fait, il semblerait que la concentration initiale en ABA dans la plante au moment de l'infection soit un élément capital pour sa fonction. Melotto et al. (2006) ont mis à profit le principe de la régulation de l'ouverture des stomates par l'ABA pour démontrer que cette hormone contrôle l'invasion bactérienne par ce mécanisme, contribuant ainsi à l'immunité de la plante. Son implication dans la synthèse de callose a de même été associée à une augmentation de la résistance à plusieurs parasites (Ton et Mauch-Mani, 2004). Le questionnement du rôle de l'ABA dans la résistance s'apparente toutefois à celui qui a été évoqué pour l'ET, eu égard à la biologie du parasitisme (biotrophe, nécrotrophe) et à l'effet dose moteur de la modulation de la signalisation. En tout état de cause, elle ne peut se dissocier des autres voies de signalisation et notamment de celles de l'ET et de l'AJ avec qui elle interagit.

\section{La coordination des voies de signalisation, vitale pour la survie de la plante}

Dans son écosystème de prédilection, une plante est sujette à des contraintes biotiques et abiotiques simultanées. Soumise à des attaques multiples, elle devra actionner l'ensemble des leviers à sa disposition pour optimiser ses stratégies de défense à moindre coût, en favorisant la communication entre les voies hormonales (crosstalk). Les approches pharmacologiques et l'utilisation des différents mutants ont révélé la multiplicité et la diversité des interconnections entre les voies de transduction du signal, traduisant les capacités adaptatives puissantes de la plante à un environnement hostile. Ces interactions peuvent avoir des effets régulateurs antagonistes ou synergiques (Koorneef et Pieterse, 2008). Un exemple est donné par l'action négative de l'AS de l'AJ et de l'ET sur l'expression des superoxydes dismutases dans le but de réguler le burst oxydatif (qui produit des formes réactives de l'oxygène) au cours de la RH. Dans certains pathosystèmes, le contrôle négatif de la voie de l'AJ passe par celle de l'AS via la protéine NPR1 cytosolique ; une mutation du gène NPR1 va activer la voie de l'AJ qui contrôle ses propres gènes de synthèse. En revanche, lors d'une attaque d'herbivore, NPR1 va inhiber la voie de l'AS pour permettre l'activation de celle de l'AJ et stimuler les réactions de défenses spécifiques de ce traumatisme mécanique. NRP1 est donc une protéine majeure dans la communication entre les voies de l'AS et de l'AJ, tout particulièrement pour son adaptation à la biologie du parasite (biotrophe versus nécrotrophe) (Spoel et al., 2007). Les mutants coil d'Arabidopsis sont altérés dans l'induction de certains mécanismes liés à l'AJ, mais surexpriment ceux dépendants de l'AS, avec une meilleure résistance aux souches virulentes de Pseudomonas syringae (Fey et al., 1994).

Les relations entre l'AS et l'ET sont, en revanche, plus obscures; des analyses de microarrays suggèrent une action potentialisatrice de l'ET sur l'induction du gène PR1 dépendant de l'AS, alors qu'une approche génétique démontre l'inverse. Ces techniques ont également révélé un effet synergique entre les voies de l'AJ et celle de l'ET dans l'induction d'un nombre de gènes de défense dans plusieurs pathosystèmes (Schenk et al., 2000), avec une action inductrice très forte pour l'ET. Chez le cotonnier infecté par Xanthomonas, la transcription du gène GhLox1 codant une 9-lipoxygénase est fortement induite par l'action combinée de ces deux hormones.

Récemment apparu sur la scène de la résistance, l'ABA interagit également avec les autres voies de signalisation. Cette molécule semble affecter la synthèse d'AJ lors de l'activation des défenses contre le champignon Pythium irregulare en intervenant en amont de ce dernier (Adie et al., 2007). En effet, les voies de l'AJ et de l'ET sont stimulées dans des mutants déficients en ABA, alors qu'un traitement de plantes avec l'ABA exogène réduit la transcription de gènes dépendants de l'AJ et de l'ET (Flors et al., 2008). Ces auteurs ont également montré que, chez Arabidopsis, le champignon nécrotrophe Alternaria brassicicola inhibe la synthèse d'ABA. Dans ce pathosystème, le gène de la callose synthase pmr4, sous contrôle de l'ABA, inhibe la voie de l'AS et permet ainsi à celle de l'AJ de contribuer la résistance à ce champignon. Un traitement par l'acide $\beta$-aminobutyrique, inducteur de résistance, mime l'effet de l'ABA en augmentant la résistance. Dans le mutant d'Arabidopsis affecté dans l'expression de pmr4, l'AS, dont l'inhibition de la voie est ainsi levée, 


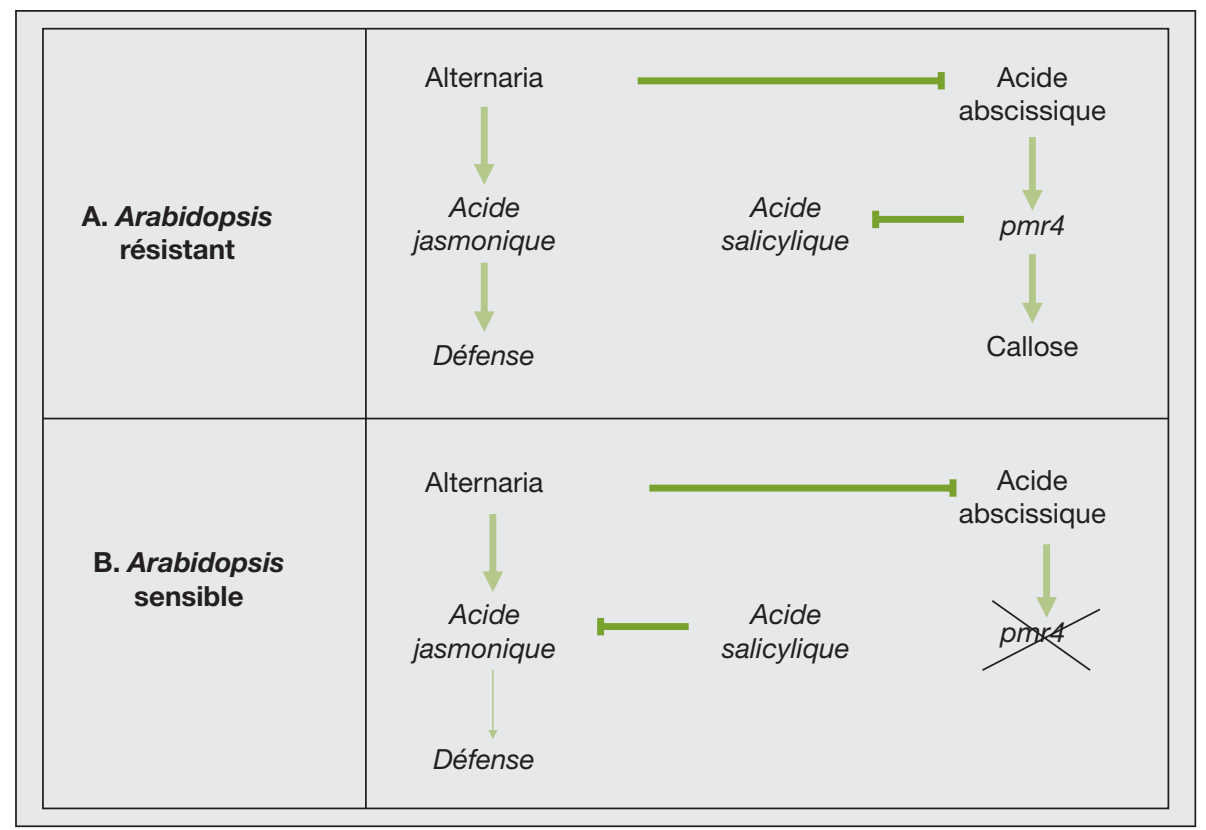

Figure 1. Signalisation de la résistance chez Arabidopsis infecté par Alternaria.

Figure 1. Signalling of Arabidopsis resistance to Alternaria.

Chez Arabidopsis, le champignon Alternaria brassicicola déclenche un mécanisme de résistance via la voie de I'acide jasmonique (A). Ce parasite réprime la synthèse d'acide abscissique ; ce dernier est cependant en quantité suffisante in planta pour induire le gène de la callose synthase (pmr4) lui-même inhibiteur de la voie de I'acide salicylique. Chez le mutant pmr4-1, affecté dans la synthèse de callose, la production d'acide salicylique va bloquer la voie de l'acide jasmonique et atténuer les réactions de défense; la plante devient sensible à A. brassicicola (B)

D'après Flors et al., 2008.

régule négativement la voie de l'AJ pour déclencher le processus de sensibilité à ce parasite. Cet exemple illustre la complexité des relations entre l'AJ, l'AS et l'ABA (figure 1) et traduit l'imbroglio de la communication entre ces voies de signalisation.

\section{Conclusion}

L'implication de la régulation hormonale dans la gestion de la résistance aux stress biotiques est déterminante dans l'issue de la confrontation entre une plante et ses agresseurs. Dans le long processus de coévolution entre les végétaux et leurs parasites, le système défensif de la plante s'est forgé une finesse de réglage qui lui permet d'adapter ses réponses pour mieux assurer sa survie. Le résultat est un équilibre trouvé par la plante entre les ressources attribuées à sa défense pour résister à l'agression parasitaire et celles nécessaires à sa croissance et sa reproduction. Dans ce scénario, l'AS, l'AJ et l'ET sont des molécules majeures, mais l'ABA ainsi que les oxylipines et les brassinostéroides contribuent tout aussi efficacement à l'expression des résistances des plantes.

Nombre de questions demeurent encore posées pour appréhender plus finement le système "immunitaire " des plantes et sa régulation. Quels sont les éléments qui vont permettre à la plante de moduler ses réponses en fonction de la biologie du micro-organisme pathogène? Comment s'effectue la régulation en fonction de la spécificité tissulaire et cellulaire? Par quels mécanismes de reconnaissance le parasite produit-il des analogues d'hormones de plante, afin d'interférer dans la régulation et de pouvoir s'alimenter tout en se protégeant des molécules microbicides produites par la plante? Dans le concert des interactions entre une plante et ses bioagresseurs, la régulation de l'action des hormones apparait donc comme un élément clé capable de faire pencher la balance, en faveur de l'un ou l'autre des protagonistes, vers la sensibilité ou la résistance (Lopez et al., 2008).

\section{Références}

Adie A, Pérez-Pérez $J$, Pérez-Pérez $M$, et al. $A B A$ is an essential signal for plant resistance to pathogens affecting JA biosynthesis and the activation of defenses in Arabidopsis. Plant Cell 2007 ; 19 : 1665-81.

Boller T. Ethylene in pathogenesis and disease resistance. In : Mattoo AK, Suttle JC, eds. The Plant Hormone Ethylene. New-York: CRC Press. 1991.

Broekaert W, Delauré S, Bolle M, et al. The role of ethylene in host-pathogens interactions. Annu Rev Phytopathol 2006 ; 44 : 393-416.

Bonaventure G, Feller A, Proebsting W, et al. A gain-of-function allele of TPC1 activates oxylipin biogenesis after leaf wounding in Arabidopsis. Plant J 2007; 49 : 889-98.

Delannoy E, Lyon B, Marmey P, et al. Resistance of cotton to Xanthomonas campestris pv. malvacearum. Annu Rev Phytopathol $2005 ; 43: 62-82$

Farmer E, Ryan C. Octadecanoid precursors of jasmonic acid activate the synthesis of woundinducible proteinase-inhibitors. Plant Cell $1992 ; 4: 129-34$.

Fey B, Benedetti C, Penfold C, et al. Arabidopsis mutants selected for resistance to the phytotoxin coronatine are male sterile, insensitive to methyl jasmonate, and resistant to a bacterial pathogen. Plant Cell 1994 ; 6 : 751-9.

Flors V, Ton J, Jakab G, et al. Abscisic acid and callose: team players in defense against pathogens? J Phytopathol $2005 ; 153$ : 1-7.

Flors $\mathrm{V}$, Ton J, van Doorn R, et al. Interplay between JA, SA and ABA signalling during basal and induced resistance against Pseudomonas syringae and Alternaria brassicola. Plant $J$ $2008 ; 54: 81-92$.

Halim V, Eschen-Lippold L, Altmann S, et al. Salicylic acid is important for basal defense of Solanum tuberosum against Phytophthora infestans. Mol Plant Microbe Interact 2007; $20: 1346-5$

Heil M, Ton J. Long distance signalling in plant defense. Trends Plant Sci 2008; 13 : 264-72.

Katsir L, Chung $\mathrm{H}$, Koo A, et al. Jasmonate signalling: a conserved mechanism of hormone. Cur Op Plant Biol 2008; 11 : 428-35.

Kazan K, Manners J. Jasmonate signaling: toward an integrated view. Plant Physio $2008 ; 146: 1459-68$.

Koorneef A, Pieterse C. Crosstalk in defense signaling. Plant Physiol 2008 ; 146 : 839-44.

van Loon L, Bakker P, Pieterse C. Systemic resistance induced by rhizosphere bacteria. Annu Rev Phytopathol 1998 ; 36 : 453-83.

van Loon L, Geraats B, Linthorst B. Ethylene as a modulator of disease resistance in plants. Trends Plant Sci 2006 ; 11 : 184-91.

Lopez M, Bannenberg G, Castresana C. Controlling hormone signalling is a plant and pathogen challenge for growth and survival. Cur Op Plant Biol 2008 ; 11 : 420-7.

Mauch-Mani B, Boller T. The role of abscisic acid in plant-pathogens interactions. Cur Op Plant Immunol 2005; 8 : 409-14.

Melotto M, Underwood W, Koczan J, et al. Plant stomata function in innate immunity against bacterial invasion. Cell 2006; 126 : 969-80.

Panter S, Jones D. Age-related resistance to plant pathogens. Adv Bot Res 2002; 38: 251-80. 
Park S, Kaimoyo E, Kumar D, et al. Methyl salicylate is a critical mobile signal for plant systemic acquired resistance. Science 2007; 318: 113-6.

Pontier D, Balague C, Roby D. The hypersensitive response A programmed cell death associated with plant resistance. CR Acad Sci III $1998 ; 321: 721-34$.

Schenk PM, Kazan K, Wilson I, et al. Coordinated plant defense responses in Arabidopisis revealed by microarrays analysis. Proc Natl Acad Sci USA $2000 ; 97$ (11) : 655-60.
Spoel S, Johnson J, Dong X. Regulation of tradeoffs between plant defenses against pathogens with different lifestyles. Proc Natl Acad Sci USA 2007 ; 104 : 18 842-7.

Stepanova A, Robertson-Hoyt G, Yun J, et al. Genes key to hormone production in plants identified. Cell 2008; 133: 177-91.

Thomma B, Penninckx I, Broekaert W, et al. The complexity of disease signaling in Arabidopsis. Cur Op Immunol 2001 ; 13 : 63-8.

Ton J, Mauch-Mani B. Beta-amino-butyric acid-induced resistance against necrotrophic pathogens is based on ABA-dependent priming for callose. Plant $J 2004 ; 38$ : 119-30.
Vallad G, Goodman R. Systemic acquired resistance and induced systemic resistance in conventional agriculture. Crop Sci 2004; 44 : 1920-34.

Verberne M, Hoekstra J, Bol JF, Linthorst $\mathrm{H}$, et al. Signaling of systemic acquired resistance in tobacco depends on ethylene perception. Plant J 2003 ; 35 : 27-32.

Vlot A, Klessig D, Park SW. Systemic acquired resistance: the elusive signal(s). Cur Op Plant Biol 2008 ; 11 : 436-42. 